behaviour $(\mathrm{p}=0.05)$, internalising problems $(\mathrm{p}=0.02)$ and critical items $(p=0.02)$. BDI scores did not differ between the groups. The VLBW group reported lower mean substance use ( $\mathrm{p}$ $=0.04$ ), mainly due to less use of alcohol. Furthermore, they reported having fewer friends, less closeness to friends, and less time spent with friends compared with controls $(p=0.05)$. When excluding 11 participants with cerebral palsy and/or low intelligence quotient ( $<2 \mathrm{SD}$ of mean in the control group), the scores for critical items, anxious/depressed and substance use were essentially the same (p-values: 0.04-0.07).

Conclusion The VLBW group reported more emotional problems than controls, and also a higher level of clinically relevant psychiatric symptoms. The findings may indicate that anxiety symptoms and a cautious lifestyle with regard to substance use are characteristics of VLBW individuals in young adulthood.

\section{PO-0826 CONOTRUNCAL HEART DEFECT IN A PATIENT WITH CONGENITAL DISORDER OF GLYCOSYLATION TYPE IA}

${ }^{1}$ A Felipe, ${ }^{2} \mathrm{DC}$ Albert, ${ }^{3} \mathrm{M}$ Girós, ${ }^{1} \mathrm{~A}$ Macaya. ${ }^{1}$ Pediatric Neurology, Hospital Universitari Vall d'Hebron, Barcelona, Spain; ${ }^{2}$ Pediatric Cardiology, Hospital Universitari Vall d'Hebron, Barcelona, Spain; ${ }^{3}$ Department of Inborn Errors of Metabolism Biochemistry and Molecular Genetics, Hospital Clínic, Barcelona, Spain

10.1136/archdischild-2014-307384.1459

Background and aims Conotruncal heart defects (CTHD) represent $15-20 \%$ of congenital heart defects; common causes are 22q11 microdelection syndrome and other chromosomal rearrangements. Congenital disorders of glycosylation (CDG) are a group of inherited multisystem disorders caused by defective glycosylation of proteins and lipids. Type I CDG is a group of heterogeneous disorders involving defective synthesis or transfer of a lipid-linked oligosaccharide precursor. The most prevalent cardiac abnormalities are cardiomyopathy and pericardial effusion, although CTHD were recently reported in two patients with CDG Ia. We describe a further case of this unusual clinical presentation.

Case report We report a 10 year-old male with neonatal diagnosis of common arterial trunk, repaired at age 17 days. Postoperative course was complicated by cardiopulmonary arrest and allegedly hypoxic ischaemic encephalopathy. He was referred to the paediatric neurology clinic for evaluation of psychomotor delay and epilepsy. Examination at age $2 y$ revealed delayed language, squint and intense hypotonia. Brain MRI revealed cerebral white matter anomalies and cerebellar atrophy, interpreted as result of his hypoxic-ischaemic event. Array-CGH and FISH for $22 \mathrm{q} 11.2$ deletion were normal. At age $8 \mathrm{y}$ he displayed ataxic gait and dysarthric speech; fat pads and inverted nipples were noted. A repeat MRI showed severe cerebellar atrophy, prompting the suspicion of CDG. Transferrin isoform analysis showed a typical CDG Ia pattern. Fibroblast phosphomannomutase activity and PMM2 mutation screen are ongoing.

Conclusions Although cardiomyopathy and pericarditis are common in CDG Ia, this condition should be suspected in CTHD, particularly when encountering unexpected neurodevelopmental delay.

\section{PO-0827 CONOTRUNCAL HEART DEFECT IN A PATIENT WITH CONGENITAL DISORDER OF GLYCOSYLATION TYPE I}

${ }^{1}$ A Felipe, ${ }^{2} \mathrm{DC}$ Albert, ${ }^{3} \mathrm{M}$ Girós, ${ }^{1} \mathrm{~A}$ Macaya. ${ }^{1}$ Pediatric Neurology, Hospital Universitari Vall d'Hebron, Barcelona, Spain; ${ }^{2}$ Pediatric Cardiology, Hospital Universitari Vall
d'Hebron, Barcelona, Spain; ${ }^{3}$ Department of Inborn Errors of Metabolism Biochemistry and Molecular Genetics, Hospital Clinic, Barcelona, Spain

\subsection{6/archdischild-2014-307384.1460}

Background and aims Conotruncal heart defects (CTHD) represent $15-20 \%$ of congenital heart defects; common causes are 22q11 microdelection syndrome and other chromosomal rearrangements. Congenital disorders of glycosylation (CDG) are a group of inherited multisystem disorders caused by defective glycosylation of proteins and lipids. Type I CDG is a group of heterogeneous disorders involving defective synthesis or transfer of a lipid-linked oligosaccharide precursor. The most prevalent cardiac abnormalities are cardiomyopathy and pericardial effusion, although CTHD were recently reported in two patients with PMM2-CDG, the most frequent CDG I. We describe a further case of this unusual clinical presentation.

Case report We report a 10 year-old male with neonatal diagnosis of common arterial trunk, repaired at age 17 days. Postoperative course was complicated by cardiopulmonary arrest and allegedly hypoxic ischaemic encephalopathy. He was referred to the paediatric neurology clinic for evaluation of psychomotor delay and epilepsy. Examination at age $2 \mathrm{y}$ revealed delayed language, squint and intense hypotonia. Brain MRI revealed cerebral white matter anomalies and cerebellar atrophy, interpreted as result of his hypoxic-ischaemic event. Array-CGH and FISH for $22 \mathrm{q} 11.2$ deletion were normal. At age $8 \mathrm{y}$ he displayed ataxic gait and dysarthric speech; fat pads and inverted nipples were noted. A repeat MRI showed severe cerebellar atrophy, prompting the suspicion of CDG. Transferrin isoforms analysis showed a typical CDG I pattern. Fibroblast phosphomannomutase activity and PMM2 mutation screen are ongoing.

Conclusions Although cardiomyopathy and pericarditis are common in CDG I, this condition should be suspected in CTHD, particularly when encountering unexpected neurodevelopmental delay.

\section{PO-0828 BONE MINERAL DENSITY AND VITAMIN D STATUS IN CHILDREN WITH CEREBRAL PALSY}

${ }^{1}$ AK Finbråten, ${ }^{2} \mathrm{U}$ Syversen, ${ }^{1} \mathrm{~J}$ Skranes, ${ }^{3} \mathrm{GL}$ Andersen, ${ }^{4} \mathrm{R}$ Stevenson, ${ }^{1} \mathrm{~T}$ Vik. ${ }^{1}$ Department of Laboratory Medicine Children's and Women's Health, Norwegian University of Science and Technology, Trondheim, Norway; ${ }^{2}$ Department of Cancer Research and Molecular Medicine, Norwegian University of Science and Technology, Trondheim, Norway; ${ }^{3}$ The Cerebral Palsy Register of Norway, Habilitation Center Vestfold Hospital, Tønsberg, Norway; ${ }^{4}$ Department of Pediatrics, University of Virginia, Charlottesville, USA

\subsection{6/archdischild-2014-307384.1461}

Background and aim Children with cerebral palsy (CP) have increased risk for low bone mineral density (BMD). The aim was to explore the difference in BMD between ambulatory and nonambulatory children with $\mathrm{CP}$ and the relationship between vitamin D status and BMD.

Methods Fifty-one children (age range: 8-18 years; 20 girls) with CP participated and had their BMD measured in the lumbar spine (LS) and the distal femur using dual X-ray absorptiometry. Children with GMFCS level I-III were defined as ambulatory ('walkers') while children with level IV-V were defined as non-ambulatory ('non-walkers'). Serum 25-hydroxyvitamin D (25-OHD) concentrations were measured as an indicator of vitamin D status.

Results Mean BMD z-score was considerably lower at the distal femur than in the LS. Non-walkers had lower mean z-scores (range: -1.7 to -5.4 ) than walkers (range: -0.8 to -1.5 ). Among 
walkers, those with GMFCS level II had lower BMD z-scores than children with level I at the distal femur ( $p$-values $<0.004$ ) but not in the LS $(p=0.06)$. Mean 25-OHD concentration was $45 \mathrm{nmol} / \mathrm{L}$ (SD: 18); lower in walkers (mean: $41 \mathrm{nmol} / \mathrm{L}$; SD: 18 ) than in non-walkers (mean: $53 \mathrm{nmol} / \mathrm{L}$; SD: 19; $\mathrm{p}=0.041$ ). There was no correlations between 25-OHD and BMD.

Conclusions The main predictor of low BMD was the inability to walk. Children with GMFCS level II had considerably lower BMD than children with level I. The majority of the CP children had insufficient vitamin D status; however, no correlation between vitamin D status and BMD was observed.

\section{PO-0829 ASSESSMENT OF BODY FAT PERCENTAGE IN CHILDREN WITH CEREBRAL PALSY}

${ }^{1}$ AK Finbråten, ${ }^{2} \mathrm{U}$ Syversen, ${ }^{1} \mathrm{~J}$ Skranes, ${ }^{3} \mathrm{G}$ Andersen, ${ }^{4} \mathrm{R}$ Stevenson, ${ }^{1} \mathrm{~T}$ Vik. ${ }^{1}$ Department of Laboratory Medicine Children's and Women's Health, Norwegian University of Science and Technology, Trondheim, Norway; ${ }^{2}$ Department of Cancer Research and Molecular Medicine, Norwegian University of Science and Technology, Trondheim, Norway; ${ }^{3}$ The Cerebral Palsy Register of Norway, Habilitation Center Vestfold Hospital, Tønsberg, Norway; ${ }^{4}$ Department of Pediatrics, University of Virginia, Charlottesville, USA

\subsection{6/archdischild-2014-307384.1462}

Background and aim Children with cerebral palsy (CP) have higher risk for malnutrition and poor growth; however, it is difficult to assess nutritional status. The aim was to assess body fat percentage based on anthropometric measurements and compare it with direct measurement of percentage body fat with dual Xray absorptiometry (DXA) in children with CP.

Methods Forty-seven children (age range: 8-18 years; 18 girls) with CP participated and had their body fat percentage measured using DXA. Body fat percentages were estimated from triceps and subscapular skinfolds using standard (Slaughter et al.) and CP-specific equations (Gurka et al.). Differences and agreement between DXA and skinfold body fat percentage were analysed by comparing mean differences by Bland-Altman plots.

Result The CP-specific equations $(r=0.883)$ and the standard equations $(r=0.819)$ had excellent correlation coefficient with DXA fat percent. The standard equations underestimated body fat percent (mean difference: $-7.1 \%$ ) measured by DXA (Figure 1). In contrast, the mean difference between fat percent

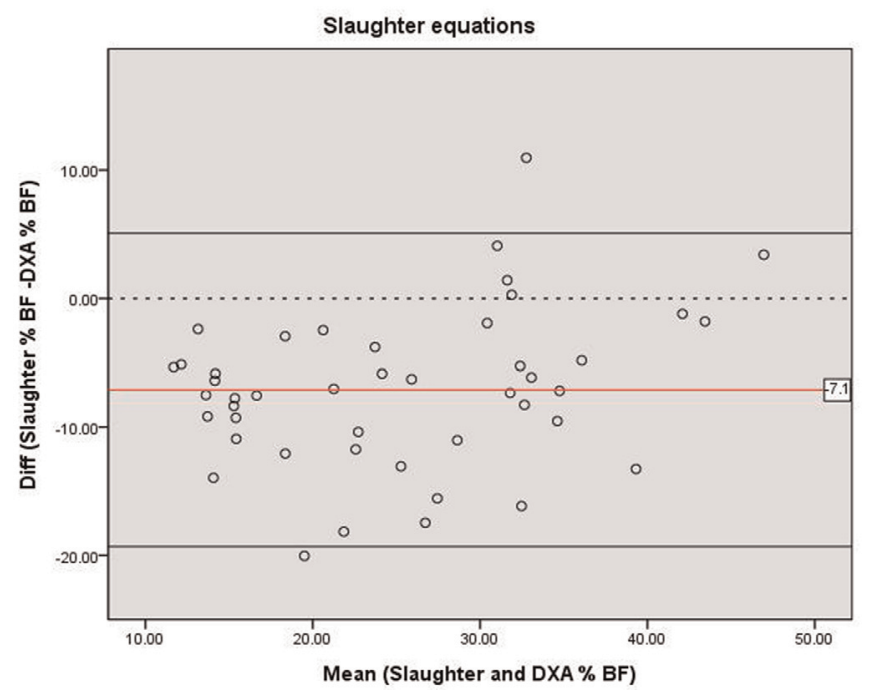

Abstract P0-0829 Figure 1

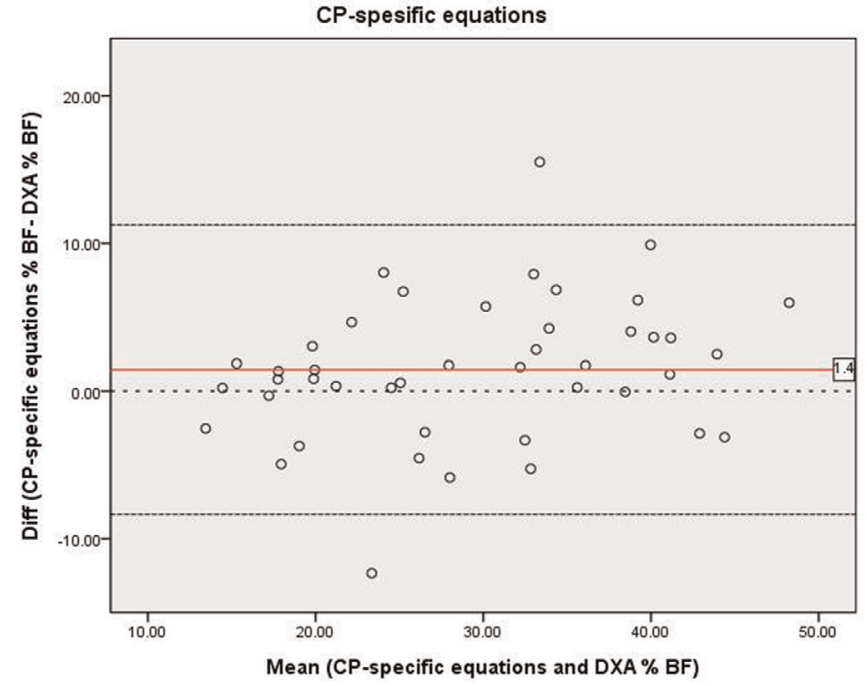

Abstract P0-0829 Figure 2

calculated by the CP-specific equations and by DXA differed marginally $(+1.4 \%)$ (Figure 2$)$.

Conclusion Accurate measures of body fat percentages may be obtained using two skinfold measurements with the CP-specific equations in children with $\mathrm{CP}$.

\section{PO-0830 IS THERE ANY CORRELATION BETWEEN POLYMORPHISM C677T METHYLENETETRAHYDROPHOLATE REDUCTASE (MTHFR) GENE AND HOMOCYSTEINE LEVEL IN CEREBRAL PALSY?}

U Gamayani. Neurology, Faculty of Medicine Universitas Padjadjaran, Bandung, Indonesia

\subsection{6/archdischild-2014-307384.1463}

Background Cerebral palsy (CP) is common cause of disability in children. The aetiology of cerebral injury in CP is multifactorial, and recent studies suggested that genetic factor maybe contributed to the development of CP. Polymorphism C677T MTHFR gene influenced homocysteine metabolism that has neurotoxic effect.

Aim This is a preliminary study. The aim of this study was to evaluate the correlation between polymorphism C677T MTHFR gene and homocysteine level in CP children in Bandung, Indonesia.

Methods This is the cross sectional study. The CP children, 414 years old were analysed C677T polymorphism in the MTHFR gene and homocysteine level. The data of this study were analysed with SPSS program, t-tests were used, and statistical significance was defined as $\mathrm{p}$ value $\leq 0,05$.

Results Thirty six spastic CP children, GMFCS I-III (22 males and 14 females; mean age 9,8 years) from school for disability children at Cibiru and Suryakanti (centre for children with special needs) in Bandung, Indonesia. There is a heterozigote polymorphism C677T MTHFR gene in 7 children $(5$ males and 2 females). The mean of homocysteine level is 8,69 . We found no significant correlation between polymorphism C677T MTHFR gene and homocysteine level in CP children $(\mathrm{p}=0,89)$

Conclusion Preliminary data shows no correlation between polymorphism C677T MTHFR gene and homocysteine level in CP 